\section{Multiples Myelom: Carfilzomib bei zytogenetischen Hochrisikopatienten}

\begin{abstract}
Die Addition des Proteasominhibitors (PI) Carfilzomib zu Lenalidomid plus Dexamethason verlängert beim rezidivierten multiplen Myelom (MM) das progressionsfreie Überleben. Gilt das auch bei Hochrisiko-Zytogenetik?
\end{abstract}

\begin{abstract}
7 ytogenetische Hochrisikovarianten $\angle$ wie die Chromosomenaberrationen $\mathrm{t}(4 ; 14)$ oder del(17p) sind bei Patienten mit MM mit einem deutlich schlechteren Überleben verbunden. In Studien mit dem ersten PI Bortezomib zeigte sich ein Nutzen bei diesen Patienten, allerdings nicht konsistent. In einer ersten Subgruppenanalyse der Phase-III-Studie ASPIRE wurde die Wirksamkeit von Carfilzomib in dieser Hochrisiko-Konstellation betrachtet. 792 Patienten mit rezidiviertem MM erhielten Lenalidomid plus Dexamethason (Rd) oder Rd plus Carfilzomib
\end{abstract}

(KRd). Beim primären Studienendpunkt progressionsfreies Überleben (PFS) erwies sich die Dreierkombination insgesamt als signifikant überlegen (median 26,3 vs. 17,6 Monate; Hazard Ratio [HR] 0,69, $95 \%$-Konfidenzintervall [95\%-KI] $0,57-0,83)$.

Die nun publizierte Subgruppenanalyse umfasste 100 Patienten mit FISHverifizierter Hochrisiko-Zytogenetik, 48 davon in der KRd- und 52 in der RdGruppe. Auch bei diesen Hochrisiko-Patienten ergab sich beim medianen PFS ein Vorteil zugunsten der Carfilzomib-
Kombination (23,1 vs. 13,9 Monate; HR 0,70, $95 \%$-KI 0,43-1,16). Bei Patienten mit zytogenetischem Standardrisiko betrug der Unterschied 10 Monate zugunsten der Carfilzomib-Gruppe. Die Ansprechraten waren in der KRd-Gruppe ebenfalls höher als in der Rd-Gruppe: Hochrisiko: 79,2 versus 59,6\% und Standardrisiko: 91,2 versus $73,5 \%$.

Die Nebenwirkungsraten waren in der Hochrisiko-Gruppe etwas erhöht, unbekannte unerwünschte Effekte traten nicht auf.

Fazit: Bei MM-Patienten mit zytogenetischem Hochrisiko verbessert die KRdKombination das PFS genauso wie bei $\mathrm{Pa}$ tienten mit Standardrisiko - jedoch nicht überproportional. Barbara Kreutzkamp

Avet-Loiseau H et al. Carfilzomib significantly improves the progression-free survival of highrisk patients in multiple myeloma. Blood. 2016; 128(9):1174-80.

\title{
Rezidiviertes MM: Carfilzomib-Triplett verbessert Lebensqualität
}

Die Lebensqualität von Patienten mit rezidiviertem multiplen Myelom unter Regimen mit neuen Substanzen wurde bisher kaum mit der unter etablierten Schemata verglichen. Nun gibt es Daten aus der ASPIRE-Studie.

$\mathrm{D}$ as Überleben von Patienten mit multiplem Myelom (MM) hat sich mit Einführung neuer Substanzen verbessert. Die Medikamente verfügen über ein spezifisches Nutzen-Risiko-Verhältnis, bei dem auch der Faktor Lebensqualität die Therapieentscheidung beeinflussen kann. In der ASPIRE-Studie wurden Wirksam-

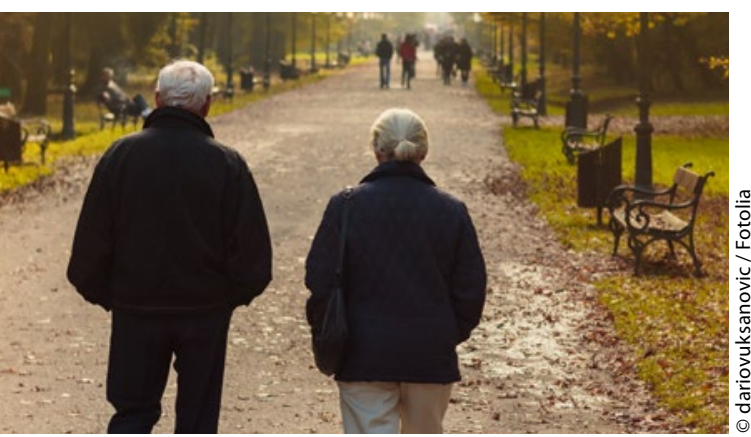

Unter KRd war der Score für den Global Health Status höher als unter Rd. keit und Sicherheit einer Kombination des Proteasominhibitors Carfilzomib mit Lenalidomid/Dexamethason (KRd) im Vergleich zu Lenalidomid/Dexamethason allein (Rd) bei Patienten mit rezidiviertem MM untersucht. Beim primären Endpunkt progressionsfreies Überleben schnitten Patienten unter der Dreierkombination signifikant besser ab. Nun liegt die Auswertung zur krankheitsspezifischen Lebensqualität als präspezifiziertem sekundärem Studienendpunkt vor.

Erfasst wurde die Lebensqualität $\mathrm{zu}$ Behandlungsbeginn, während und am Ende der Therapie mithilfe zweier EORTC-Fragebögen (QLQ-C30, QLQMY20). Über die 18 Behandlungszyklen gaben die Patienten der KRd-Gruppe insgesamt einen höheren Score in der Global Health Status/Quality of Life(GHS/QoL)Skala an als die der Kontrollgruppe $(p<0,001)$. Der als minimale erforderliche Differenz definierte Unterschied von
5 Skalenpunkten zwischen beiden Behandlungsgruppen wurde in Zyklus 12 erreicht (5,6 Punkte). Keine Unterschiede zwischen beiden Gruppen bestanden bei anderen Subskalen wie Fatigue, Übelkeit, Erbrechen, Schmerzen, physische Funktionsfähigkeit, Rollenfunktion, Krankheitssymptome und Nebenwirkungen. Die für ein GHS/QoL-Ansprechen prädefinierte Zunahme von $\geq 5$ Punkten auf der GHS/QoL-Skala pro Zyklus wurde von mehr KRd-Patienten erreicht - mit statistisch signifikanten Unterschieden in Zyklus 12 und 18 (25,5 vs. $17,4 \%$; $\mathrm{p}<0,01$ und 24,2 vs. $12,9 \%$; $<<0,001)$.

Fazit: Carfilzomib zusätzlich zu Rd verbesserte in einem Subgruppen-Vergleich die Lebensqualität von Patienten mit rezidiviertem MM im Vergleich zu Rd alleine leicht.

Barbara Kreutzkamp

Stewart AK et al. Health-related quality of life results from the open-label, randomized, phase III ASPIRE trial evaluating carfilzomib, lenalidomide, and dexamethasone versus lenalidomide and dexamethasone in patients with relapsed multiple myeloma. J Clin Oncol. 2016 Sep 6.

[Epub ahead of print] 